\title{
Dynamics of circulating tumor cells in early breast cancer under neoadjuvant therapy
}

\author{
MARÍA JOSÉ SERRANO ${ }^{1}$, PEDRO SÁNCHEZ ROVIRA², I. MARTÍNEZ-ZUBIAURRE ${ }^{3}$, \\ MIGUEL DELGADO RODRIGUEZ ${ }^{4}$, MÓNICA FERNÁNDEZ ${ }^{2}$ and JOSE A. LORENTE ${ }^{1,5}$
}

\author{
${ }^{1}$ Centro Pfizer, Universidad de Granada, Junta de Andalucía de Genómica e Investigación Oncológica (GENYO), Granada; \\ ${ }^{2}$ Medical Oncology Department, University Hospital, Jaén, Spain; ${ }^{3}$ Institute of Clinical Medicine, University of Tromso, \\ Tromso, Norway; ${ }^{4}$ Department of Health Sciences, Faculty of Experimental Sciences, University of Jaén, Jaén; \\ ${ }^{5}$ Laboratory of Genetic Identification - UGR, Department of Legal Medicine, University of Granada, Granada, Spain
}

Received November 4, 2011; Accepted January 20, 2012

DOI: $10.3892 / \mathrm{etm} .2012 .540$

\begin{abstract}
At present, the majority of patients with breast cancer are diagnosed at early stages of disease development. However, a considerable number of such cases develop secondary malignancies after a relatively short period of time. The presence of circulating tumor cells (CTCs) has been proposed as a strong biomarker to predict disease recurrence in metastatic breast cancer. However, the prognostic significance is not clear in early breast cancer. We present results on CTC determination in peripheral blood in non-metastatic breast cancer patients in the context of neoadjuvant treatment. Twenty-six breast cancer patients, scheduled for neoadjuvant therapy, were enrolled in a prospective study, of which 24 were able to complete therapy. CTC assessment was performed by sorting out cytokeratin-positive cells from $10 \mathrm{ml}$ of peripheral blood using immunomagnetic separation, followed by immunocytochemical characterization of cells. Seventeen blood samples out of 24 patients were CTC-positive when collected prior to neoadjuvant chemotherapy. No significant correlations were found between the presence of CTCs and lymph node status $(\mathrm{p}=0.1)$, histological type $(\mathrm{p}=0.802)$, stage $(\mathrm{p}=0.43)$ or overall survival (OS) $(\mathrm{p}=0.599)$. Thirteen CTC-positive samples were observed in blood samples collected after treatment. Univariate analyses revealed that the presence of CTCs was related to OS when the detection was positive both before and after treatment $(\mathrm{p}=0.023)$. CTCs can be a strong
\end{abstract}

Correspondence to: Dr María José Serrano, Centro Pfizer, Universidad de Granada, Junta de Andalucía de Genómica e Investigación Oncológica (GENYO), Health Sciences Technologic Park, Av. de la Ilustración, 114, ES-18007 Granada, Spain

E-mail: mjose.serrano@genyo.es

Abbreviations: CTCs, circulating tumor cells; ER, estrogen receptor; CK, cytokeratin; NAT, neoadjuvant therapy; OS, overall survival

Key words: circulating tumor cells, overall survival, neoadjuvant therapy, early breast cancer prognostic marker in early breast cancer. The persistence of CTCs before and after treatment can identify a subpopulation of patients with an increased risk of recurrence.

\section{Introduction}

Historically, most cancer research has been focused on studying the biology of either primary tumors or metastases. However, the intermediate steps of the process, including events such as cell departure from the tumor mass, intravasation, lymphatic and circulatory dissemination and extravasation, have been less studied. In recent years, there has been an increasing interest in understanding thoroughly all processes involved in the metastatic cascade, including the transit journey of tumor cells in the circulatory and lymphatic systems (1). Moreover, it is acknowledged that a thorough understanding of the biology of circulating tumor cells (CTCs) may open new paths for the future development of potential anticancer strategies $(2,3)$.

The systemic nature of breast cancer is characterized by the migration of tumor cells even at early stages of the disease when the primary tumor shows a relatively small size (4). Thus, CTCs in peripheral blood could be regarded as the pre-stadium of clinically manifest distant metastases $(5,6)$. A considerable number of studies have been accomplished on the determination of CTCs as a prognostic and/or predictive biomarker for different types of cancers (7). In contrast to studies in bone marrow (BM), only a few studies on peripheral blood (PB) screening have been conducted to date $(8,9)$. Additionally, the size of the patient cohorts analyzed and the applied detection methods vary considerably between these studies. Such experimental and analytical diversity insinuates that the available knowledge on this topic is in certain cases contradictory and controversial. Consequently, although some groups have found an association between the presence of CTCs and a worse prognosis, others have not demonstrated such an association, making the prognostic significance of CTCs still uncertain $(10,11)$.

For most patients affected with primary breast cancer, the standard of care is systemic neoadjuvant therapy (NAT), followed by surgical resection of the malignant tissue. NAT may result in local tumor regression or even in a complete 
tumor response, which may directly influence the surgical procedure of choice, going from radical mastectomy to some type of breast-conserving surgery - without risking patient survival (12). Even though at early stages tumors are clinically restricted to loco-regional tissue, there is often early dissemination of viable tumor cells. One of the purposes of systemic NAT is to attack these circulating and/or disseminating tumor cells. This fact has potentiated the interest in the use of NAT (13).

A potential use of the detection CTCs could be to optimize treatment strategies that may increase the cure rate of breast cancer patients. Several studies have reported that metastatic breast cancer patients (MBC) with decreasing CTCs after the initiation of antitumor therapy tend to enjoy a prolonged benefit from their current regimens (14). In early breast cancer, however, it remains necessary to consuct more studies to correlate the presence or persistence of CTCs with overall survival (OS) and with progression-free disease (PFS). We designed a study to assess the potential of CTCs to predict risk of death in a cohort of patients with primary breast cancer, evaluating the presence of CTCs both prior to and after neoadjuvant treatment.

\section{Patients and methods}

Patient characteristics. Twenty-six patients were enrolled in the study from April 2000 until December 2002, but 2 patients were excluded due to problems originating during the sample processing. Follow-up time was extended until the end of 2005 or until death, with a median follow-up time of 56 months. Eligibility criteria for the study were female breast cancer with age ranging from 26 to 71 years, with early breast cancer (stages T2 to T3), without a familial history of cancer and without previous chemotherapy treatment at the entry of the study. Tumor types of the patients were classified by pathologists according to the World Health Organization scheme for typing breast tumors. Immunostaining of primary tumor samples was assessed by the Department of Pathology (University Hospital of Jaén) on routinely processed paraffin sections upon antigen-retrieval. Sections were examined with monoclonal antibodies (mAbs) recognizing estrogen receptor (ER) (clone 6F11; Master Diagnostica, Granada, Spain); and HER2 c-erbB2 (clone CB11; Master Diagnostica), using immunoperoxidase staining with monoclonal antibodies, and considering the ER positive cut-off level at $>10 \%$ stained nuclei. The receptor status of the patients was $59 \%$ for positive ER and $41 \%$ for negative ER. The clinical characteristics of the patients at the time of primary diagnosis are outlined in Table I.

Neoadjuvant chemotherapy. All of the patients received anthracyclines and taxol followed by tamoxifen aromatase inhibitors for patients with hormone receptor-positive tumors. All evaluations were performed with informed consent of the patients and with no knowledge of the patients' clinical status.

Circulating tumor cell detection. Prior to the first cycle of chemotherapy and 1 month after the end of the treatment, $10 \mathrm{ml}$ of anti-coagulated PB were collected. Samples were maintained at room temperature and processed within $4 \mathrm{~h}$ after collection. Blood samples were processed according to the protocol established for our group (15). Briefly, the samples were processed initially in a double density gradient (Histopaque 1077 over Histopaque 1119). Enrichment of epithelial cells after Ficoll gradient was performed by selective immunomagnetic cell separation, using magnetic beads labelled with a multi-cytokeratin-specific antibody (CK3-11D5; Miltenyi Biotec) which recognizes cytokeratin 7, 8, 18 and 19. Cytokeratin-positive cells were further characterized by immunocytochemical staining using a highly specific anti-cytokeratin-FITC antibody (CK3-6H5-FITC; Miltenyi Biotec), on slides prepared by cytospin. Additionally, cytokeratin-expressing cells were revealed by incubation with freshly prepared Fast Red TR/Naphthol AS-MX substrate solution (Sigma) for $15 \mathrm{~min}$ in a humidity chamber at room temperature. Slides were washed once with PBS and stained with Mayer's hematoxylin solution (Sigma) for $30 \mathrm{sec}$ at room temperature. Epithelial tumor cells can quickly and easily be identified and enumerated based on their strong coloration. The efficiency of the method was tested using positive controls prepared by spiking blood samples of healthy volunteers with varying numbers of MDA-MB-231 and MCF-7 mammary epithelial cancer cell lines (15).

Statistical analysis. The clinical characteristics of the patients were related to the presence of $\mathrm{CK}^{+}$cells in $\mathrm{PB}$ by means of a Fisher's exact test. When a variable was ordered, a trend analysis was applied. Actuarial curves for OS were calculated by the Kaplan-Meier method. The magnitude of the association was assessed in terms of hazard ratios with $95 \%$ confidence intervals (CI), estimated by a Cox regression analysis. OS was calculated from the date of the detection of CK cells in PB to the date of death. All statistical calculations were carried out using the STATA 8-SE (Stata Inc., College Station, TX, USA) statistical analysis program. The cut-off point for significance was set as $\mathrm{p}<0.05$.

\section{Results}

From April 2000 until December 2002, pre- and postneoadjuvant chemotherapy blood samples were obtained from 24 patients. CTCs detected by immunocytochemistry in the $\mathrm{PB}$ of breast cancer patients showed a strong cytoplasmic staining pattern, and the surrounding hematopoietic cells showed no expression of cytokeratins (Fig. 1). Identification of at least $1 \mathrm{CTC}$ in the sample was considered as positive. Correlation of CTCs in PB with patient characteristics is outlined in Table I.

In the samples collected prior to neoadjuvant chemotherapy, one or more CTCs were detected in 17 patients (detection rate $70.08 \%$; $95 \%$ CI $0.11-2.33$ ). The mean (SD) number of $\mathrm{CK}^{+}$cells detected was $4.3( \pm 6.3)$, ranging from 1 to 28 cells (Tables I and II). There were significant differences between the presence of $\mathrm{CK}^{+}$cells and age groups $(\mathrm{p}=0.048)$, in favor of patients $<50$ years of age. No correlations were found with lymph node status $(\mathrm{p}=0.1)$, histological type $(\mathrm{p}=0.802)$ or tumor stage $(p=0.43)$. Regarding the relationship between ER and the presence of CTCs, a borderline, but significant trend was observed $(\mathrm{p}=0.093)$. Univariate analyses, including all 
Table I. Tumor characteristics for the studied population and correlation with CTC detection prior to and after neoadjuvant chemotherapy.

\begin{tabular}{|c|c|c|c|c|c|c|}
\hline & \multicolumn{3}{|c|}{ Basal status $(n=24)$} & \multicolumn{3}{|c|}{ Post-chemotherapy $(n=24)$} \\
\hline & No. $(\%)$ & $\mathrm{CTCs}^{+}(\mathrm{n}=17)$ & p-value & No. $(\%)$ & $\mathrm{CTCs}^{+}(\mathrm{n}=13)$ & p-value \\
\hline Age (years; 55.38$)^{\mathrm{a}}$ & & & $0.048^{\mathrm{a}}$ & & & 0.500 \\
\hline$<50$ & $9 / 24(37.5)$ & 8 & & $8 / 24(33)$ & 5 & \\
\hline$\geq 50$ & $15 / 24(62.5)$ & 9 & & $16 / 24(67)$ & 8 & \\
\hline Stage & & & 0.430 & & & 0.240 \\
\hline IIA-IIB & 9/23 (39.1) & 8 & & $9 / 24(37.5)$ & 6 & \\
\hline IIIA-IIIB & $14 / 23(60.9)$ & 8 & & $15 / 24(62.5)$ & 7 & \\
\hline Histology & & & 0.802 & & & 0.600 \\
\hline Ductal & $11 / 23(48)$ & 8 & & $12 / 23(52.2)$ & 7 & \\
\hline Lobular & $6 / 23(26)$ & 4 & & $5 / 23(21.7)$ & 3 & \\
\hline Other & $6 / 23(26)$ & 4 & & $6 / 23(26.1)$ & 2 & \\
\hline Lymph node status (N) & & & 0.100 & & & 0.150 \\
\hline No & $7 / 23(30.4)$ & 6 & & $3 / 21(14)$ & 1 & \\
\hline $\mathrm{N} 1$ & $11 / 23(47.9)$ & 7 & & $13 / 21(62)$ & 7 & \\
\hline $\mathrm{N} 2$ & $5 / 23(21.7)$ & 3 & & $5 / 21(24)$ & 2 & \\
\hline Estrogen receptor (ER) & & & $0.093^{\mathrm{b}}$ & & & $0.097^{\mathrm{b}}$ \\
\hline ER-positive & $13 / 22(59)$ & 11 & & $13 / 22(59.1)$ & 9 & \\
\hline ER-negative & $9 / 22(41)$ & 4 & & $9 / 22(40.9)$ & 2 & \\
\hline
\end{tabular}

$\mathrm{CTCs}^{+}$, number of patients with CK-positive cells. ${ }^{a}$ Satistically significant; ${ }^{\text {b }}$ borderline significant.

Table II. Number of CTCs detected in 24 breast cancer patients.

\begin{tabular}{|c|c|c|c|}
\hline Patient & $\begin{array}{c}\text { CTCs basal } \\
\text { status }\end{array}$ & $\begin{array}{l}\text { CTCs post- } \\
\text { chemotherapy }\end{array}$ & $\begin{array}{l}\text { Patient } \\
\text { status }\end{array}$ \\
\hline 1 & 14 & 2 & Exitus \\
\hline 2 & 6 & 3 & Exitus \\
\hline 3 & 0 & 1 & Alive \\
\hline 4 & 0 & 0 & Exitus \\
\hline 5 & 2 & 0 & Alive \\
\hline 6 & 28 & 1 & Exitus \\
\hline 7 & 2 & 0 & Alive \\
\hline 8 & 0 & 8 & Alive \\
\hline 9 & 0 & 1 & Alive \\
\hline 10 & 0 & 3 & Alive \\
\hline 11 & 7 & 0 & Alive \\
\hline 12 & 1 & 0 & Alive \\
\hline 13 & 6 & 2 & Exitus \\
\hline 14 & 3 & 0 & Alive \\
\hline 15 & 2 & 0 & Alive \\
\hline 16 & 3 & 1 & Exitus \\
\hline 17 & 3 & 0 & $\begin{array}{l}\text { Alive with metatastic } \\
\text { disease }\end{array}$ \\
\hline 18 & 2 & 0 & $\begin{array}{l}\text { Alive with metatastic } \\
\text { disease }\end{array}$ \\
\hline 19 & 0 & 8 & Alive \\
\hline 20 & 15 & 6 & Exitus \\
\hline 21 & 0 & 0 & Alive \\
\hline 22 & 0 & 8 & Alive \\
\hline 23 & 8 & 0 & Alive \\
\hline 24 & 6 & 0 & Alive \\
\hline
\end{tabular}

CTCs were counted prior to and post NAT. Clinical evolution of the patients during follow-up. patients, revealed that the presence of CTCs was not related to the OS ( $\mathrm{p}=0.599)$.

In the post-neoadjuvant chemotherapy sample group, 13 out of 24 patients were CTC-positive (detection rate 54.1\%; range 1-8 cells, median 2.1) (Tables I and II). Comparing the rate of CTCs present in samples from patients prior to and after NAT, persistent detection of CTCs was achieved in 6 of 24 patients (Fig. 2).

There were no significant differences in the presence of $\mathrm{CK}^{+}$cells between the age groups $(\mathrm{p}=0.5)$, the lymph node status $(p=0.15)$ or histological type $(p=0.6)$ (Table I) when compared to the presence of CTC at both pre- and postNAT. In regards to the ER, a borderline significant trend was observed $(\mathrm{p}=0.097)$.

Prognostic analysis. Kaplan-Meier analysis of the OS in connection with the detection of $\mathrm{CK}^{+}$cells is presented in Fig. 3. Follow-up time covered the period between last sample collection and the end time-point of the study or until the date of death (median follow-up 56 months). Complete data were available from 24 patients. From the initial 24 patients included in the study, 7 patients had disease progression despite treatment and died within the follow-up time. Within this group, 6 of them presented persistence of CTCs during follow-up and 1 of them was negative for CTCs. Patients with negative result for CTCs, in any of the excisions obtained during the follow-up, remain alive and without evidence of clinical disease, except 1 individual. Univariate and multivariate analyses revealed that the presence of CTCs was related to OS, but only when the detection of CTC was positive prior to and after treatment. Hence, only in the double-positive patients can CTC detection be regarded as an independent prognostic factor. Likewise, the 


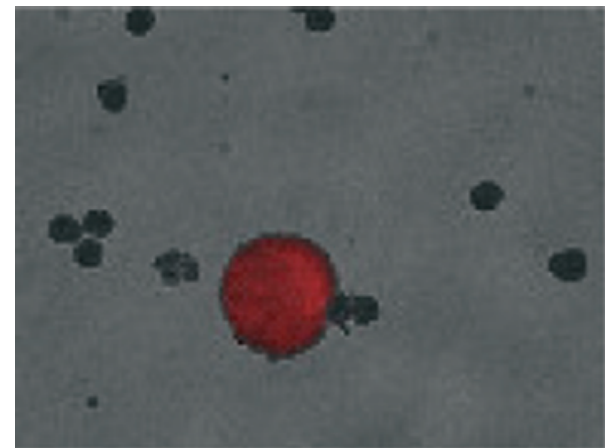

Figure 1. Circulating tumor cell detected in a breast cancer patient after neoadjuvant therapy.

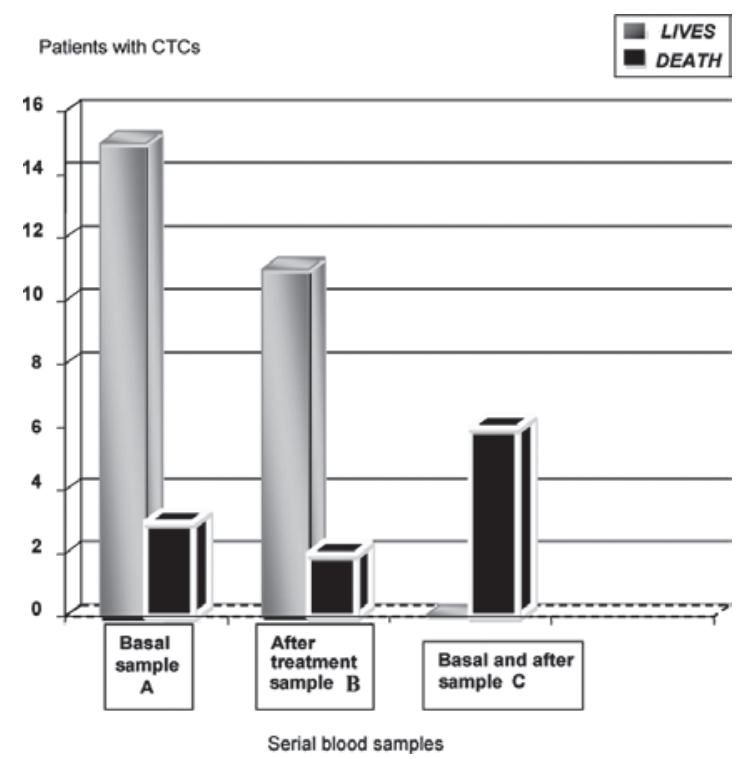

Figure 2. Association between persistence of circulating tumor cells (CTCs) and mortality in breast cancer patients with neoadjuvant therapy. Grey, number of patients that are alive and exhibiting CTCs; Black, number of patients deceased and exhibiting CTCs. (A) No. of patients in basal status with CTCs. (B) No. of patients after treatment with CTCs. (C) No. of patients with CTCs prior to and after treatment.

Kaplan-Meier survival analysis showed that the persistence of $\mathrm{CK}^{+}$cells in PB was associated with short survival $(\mathrm{p}=0023)$.

\section{Discussion}

Mounting evidence during recent years suggests that the presence of CTCs correlates with disease progression in patients with breast cancer (16). The prognostic value of CTCs has been validated in MBC, but the clinical significance of CTCs at early stages of breast cancer remains controversial. In the present study, we demonstrated a significant correlation between CTC detection and disease progression when the CTC count was positive ( $\geq 1$ cells $/ 10 \mathrm{ml}$ blood) at both pre- and post-neoadjuvant treatment.

Similar studies to ours have been previously conducted In a recent report by Bidard et al (17), detection of CTCs in non-MBC patients was correlated with OS when neoadjuvant chemotherapy was chosen as a treatment modality. In that study, after applying CellSearch system for CTC separation,

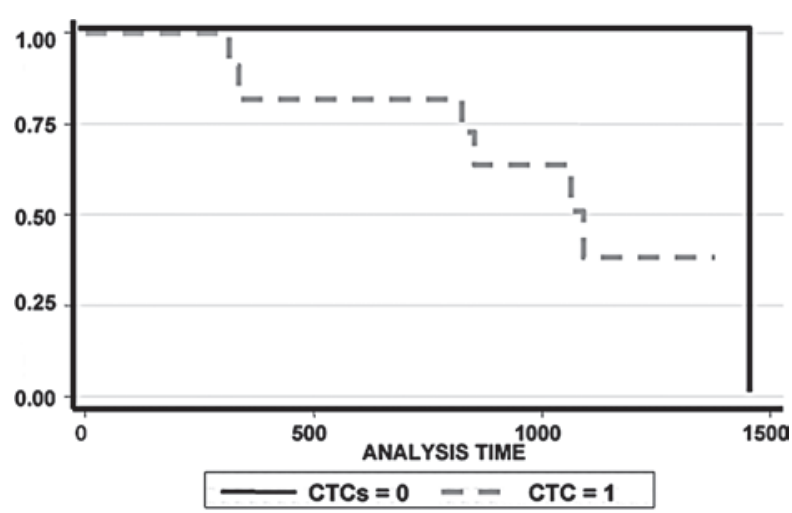

Log-rank test for equality of survivor functions

\begin{tabular}{|l|l|l|}
\hline CTC & Events Observed & Events expected \\
\hline 0 & 1 & 4.64 \\
\hline 1 & 6 & 2.36 \\
\hline TOTAL & 7 & 7.00 \\
\hline \\
Chi2(1) $=9.36$ \\
Prochi2 $=0.0023$
\end{tabular}

Figure 3. Kaplan-Meier analysis of the overall survival in connection with the detection of $\mathrm{CK}^{+}$cells showing that only patients with the persistence of $\mathrm{CK}^{+}$cells in peripheral blood were associated with short survival.

the group reported that detection of 1 or more CTCs/7.5 ml prior to NAT predicted OS. Here, we reinforced the notion that the presence of CTCs in the neoadjuvant context can be used as a prognostic factor when 1 or more cells are detected in PB. However, in our study this clinical significance was only valid when a persistent detection of CTCs after treatment occurred. Only patients with remaining CTCs in the blood after treatment clearly showed a shorter OS. Similar to our findings, the authors showed that in multivariate analysis the presence of CTCs after NAT was found to be of less significance for OS. Contrary to our data, their results for OS demonstrated that the presence of CTCs before chemotherapy was a strong independent prognostic factor along with tumor size and triple receptor-negative phenotype.

Other groups have also made attempts to explore the prognostic value of CTC detection in the context of breast cancer. Riethdorf et al in the GepartQuatro study observed no significant correlation between CTC detection and primary tumor characteristics, such as tumor stage, histologic type, node lymph stage or homone receptor status (18). Comparable outcomes were shown by Pierga et al (9) in a smaller cohort of patients in the REMAGUS 02 trial. In this study, we also observed no significant correlation between CTC detection and most characteristics presented in the primary tumor. To note, a close to significant correlation between CTC detection and ER status was observed in our study $(\mathrm{p}=0.097)$, which could be relevant considering that we presented results with a smaller cohort of patients.

The potential of CTCs to accurately predict the risk of relapse and OS may depend on the optimal timing for sampling, the frequency of performing blood collection and 
the cell separation system used. The positive immunomagnetic isolation used here was performed using mAb-labelled magnetic microbeads and a basic magnet (MACS ${ }^{\mathrm{TM}}$ magnetic activated cell sorting system-Miltenyi Biotec). This methodology allows efficient sorting of CTCs from leukocytes. In previous reports from our laboratory, we demonstrated that this methodology holds high reproducibility and accuracy (19). In fact, CellSearch ${ }^{\mathrm{TM}}$ system (Veridex) is the only FDA approved and leading automated immnunomagnetic separation system for clinical routines to detect and analyze CTCs in patients with MBC. Nevertheless, the efficacy of this system in samples collected from early breast cancer patients still needs to be confirmed. Other important points for consideration are the timing of sample collection and the definition of positivity in regard to the number of cells. Cristofanilli et al observed that in MBC, detection of CTCs at relative high numbers $(\geq 5)$ at any subsequent time point ( 3 weeks onwards) appears to be an indicator of poor clinical outcome, and they concluded that determinations of CTCs up to 15-20 weeks after initiation of therapy predicts more discriminately for survival outcome. In their study, patients showing a persistence of CTC counts ( $\geq 5$ CTCs at the beginning and follow-up) exhibited significantly worse OS compared to patients with persistently low CTC counts ( $<5$ at baseline and follow-up). On the other hand, patients who changed from an elevated CTC count $(\geq 5)$ at baseline to a low CTC count $(<5)$ at follow-up survived significantly longer than those patients who changed from a low baseline CTC count to an elevated CTC count at follow-up (20). However, such correlations are not as evident in early breast cancer. In this regard, Riethdorf et al (18) established CTC positivity at 1 or more CTCs/7.5 ml before treatment, while analyzing blood samples from pre-operated non-metastatic breast cancer patients. In that study, only $5 \%$ of pre-operative samples carried 5 or more CTCs/sample. In our study, we observed that the majority of patients with fatal outcome presented 5 or more CTCs/10 ml prior to NAT. However, the numbers of cells in positive samples in average diminished considerably in blood collected after treatment. Still, our results were obtained from a small cohort of patients; therefore interpretation of the data should be carried out with caution.

We did not find correlations between CTCs and the pathological characteristics of tumors at diagnosis. In early breast cancer cases, Pachman et al (21) suggested the existence of a strong correlation between the presence of CTCs and a decrease in tumor size after neoadjuvant chemotherapy. The authors theorized that the reduction in the tumor size during treatment could be a consequence of the release of CTCs from the primary tumor mass. Moreover, another study revealed that escalating numbers of CTCs during tamoxifen treatment, although associated to primary tumor mass reduction, was a strong predictor of relapse (22). In this later study, the observed enhancement in CTC positivity was also predictive of a subsequent relapse after aromatase inhibitor treatment. Additionally, other groups exploring similar associations also found an inverse correlation between CK-19 mRNA positivity in samples pre- and post-tamoxifen treatment and disease-free interval and OS (23). The overall conclusion of all these observations is that, in most cases, the CTCs found after treatments are refractory to therapy. We hypothesize that these CTCs could represent poorly differentiated cells. A reasonable possibility is that NAT may eliminate more efficiently highly differentiated tumor cells, and less efficiently the poorly differentiated CTCs or/and in a dormant state (24). Experiments in pre-clinical models suggest that dormant cancer cells are common in that they may get reactivated under specific circumstances, and that they represent an interesting but difficult therapeutic target (25).

In summary, we show that CTC determination in early breast cancer patients can be used as a biomarker for prognosis when the detection persists several weeks after treatment. This methodology, applied at the parameters that we have chosen, serves to identify patients with a high risk of death after treatment. Our data also suggest that most CTCs found after treatment may represent poorly differentiated cells refractory to treatment, since it is associated with a higher degree of relapse. Although significant, the power of our analyses is limited by the number of patients enrolled in the study, despite the relatively long follow-up period. Furthermore, the potential loss of epithelial markers suffered by tumor cells released to the circulation or by tumor cells going through partial or full epithelial-mesenchymal transdifferentiation may lead to erroneous outcomes and still stands as one of the main drawbacks of this methodology.

Standardization of the methodology between laboratories remains as a mandatory goal to reach a sound communication and the universal acceptance of data among scholars in the field.

Traslational relevance. In recent years, the perspective of the study of breast cancer has changed considerably, and the importance of curative local treatments has declined in favor of administering chemotherapy treatments or hormonal, even in the absence of lymph node involvement or small-size tumors. The identification of new prognostic factors that allow us to discriminate and stratify patients for individualized treatment is one of the most important avenues of research in this field. Among these new markers, the presence of CTCs is considered indicative of hematogenous spread. A factor with considerable potential prognostic significance in metastasis formation could allow the characterization of patients with primary breast cancer into subgroups that require more intensive clinical monitoring. Lastly, with molecular and genetic characterization of CTCs, chemoresistance profiles should also be able to advise the clinician regarding the most efficacious chemotherapy regimens. In terms of tumor biology, it is clear that circulating tumor cells are present in early breast cancer, thus supporting the theory of early metastasis.

\section{Acknowledgements}

The authors gratefully acknowledge all patients who took part in this study and would like to thank Miguel Rivero, Jesus Ruiz, Ivan Álvarez and Carlos García (Miltenyi Biotec Spain) for the unconditional support in cancer research. The study was supported by the Ministerio de Sanidad y Consumo grant; grant no. FIS 02/1879; grant sponsor: COFIMAN S.L. and Fundación Pública Progreso y Salud, Consejería y Salud, Junta de Andalucía. 


\section{References}

1. Klein CA: The metastasis cascade. Science 321: 1841-1844, 2008.

2. Mego M, Mani SA and Cristofanilli M: Molecular mechanisms of metastasis in breast cancer - clinical applications. Nat Rev Clin Oncol 7: 693-701, 2010.

3. Graves $\mathrm{H}$ and Czerniecki BJ: Circulating tumor cells in breast cancer patients: an evolving role in patient prognosis and disease progression. Pathol Res Int 2011: 621090, 2011.

4. Pantel K and Brakenhoff RH: Dissecting the metastatic cascade. Nat Rev Cancer 4: 448-456, 2004.

5. Serrano MJ, Sánchez-Rovira P, Delgado-Rodriguez M and Gaforio JJ: Detection of circulating tumor cells in the context of treatment: prognostic value in breast cancer. Cancer Biol Ther 8: 671-675, 2009 .

6. Botteri E, Sandri MT, Bagnardi V, Munzone E, Zorzino L, Rotmensz N, Casadio C, Cassatella MC, Esposito A, Curigliano G et al: Modeling the relationship between circulating tumour cell number and prognosis of metastatic breast cancer. Breast Cancer Res Treat 122: 211-217, 2010

7. Lin H, Balic M, Zheng S, Datar R and Cote R: Disseminated and circulating tumor cells: role in effective cancer management. Crit Rev Oncol Hematol 77: 1-11, 2011.

8. García-Sáenz JA, Martín M, Maestro ML, Vidaurreta M, Veganzones S, Rafael S, Casado A, Bobokova J, Sastre J, de la Orden V, et al: Circulating tumour cells in locally advanced breast cancer. Clin Transl Oncol 11: 544-547, 2009.

9. Pierga JY, Bidard FC, Mathiot C, Brain E, Delaloge S, Giachetti S, de Cremoux P, Salmon R, Vincent-Salomon A and Marty M: Circulating tumor cell detection predicts early metastatic relapse after neoadjuvant chemotherapy in large operable and locally advanced breast cancer in a phase II randomized trial. Clin Cancer Res 14: 7004-7010, 2008.

10. Nakagawa T, Martinez SR, Goto Y, Koyanagi K, Kitago M, Shingai T, Elashoff DA, Ye X, Singer FR, Giuliano AE and Hoon DSB: Detection of circulating tumor cells in early-stage breast cancer metastasis to axillary lymph nodes. Clin Cancer Res 13: 4105-4110, 2007.

11. Wong NS, Kahn HJ, Zhang L, Oldfield S, Yang LY, Marks A and Trudeau ME: Prognostic significance of circulating tumour cells enumerated after filtration enrichment in early and metastatic breast cancer patients. Breast Cancer Res Treat 99: 63-69, 2006.

12. Langer R, Ott K, Feith M, Lordick F, Siewert JR and Becker K: Prognostic significance of histopathological tumor regression after neoadjuvant chemotherapy in esophageal adenocarcinomas. Mod Pathol 22: 1555-1563, 2009.

13. Makhoul I and Kiwan E: Neoadjuvant systemic treatment of breast cancer. J Surg Oncol 103: 348-357, 2011.

14. Pierga JY, Hajage D, Bachelot T, Delaloge S, Brain E, Campone M, Dieras V, Rolland E, Mignot L, Mathiot C and Bidard FC: High independent prognostic and predictive value of circulating tumor cells compared with serum tumor markers in a large prospective trial in first-line chemotherapy for metastatic breast cancer patients. Ann Oncol: 2011. doi:10.1093/annonc/mdr263, 2011
15. Riethdorf S and Pantel K: Disseminated tumor cells in bone marrow and circulating tumor cells in blood of breast cancer patients: current state of detection and characterization. Pathobiology 75: 140-148, 2008.

16. Gaforio JJ, Serrano MJ, Sanchez-Rovira P, Sirvent A, Delgado-Rodriguez M, Campos M, de la Torre N, Algarra I, Duenas R and Lozano A: Detection of breast cancer cells in the peripheral blood is positively correlated with estrogen-receptor status and predicts for poor prognosis. Int J Cancer 107: 984-990, 2003.

17. Bidard FC, Mathiot C, Delaloge S, Brain E, Giachetti S, de Cremoux P, Marty M and Pierga JY: Single circulating tumor cell detection and overall survival in nonmetastatic breast cancer. Ann Oncol 21: 729-733, 2010.

18. Riethdorf S, Müller V, Zhang L, Rau T, Komor M, Roller M, Huober J, Fehm T, Schrader I, Hilfrich J, et al: Detection and HER2 expression of circulating tumor cells: prospective monitoring in breast cancer patients treated in the neoadjuvant geparquattro trial. Clin Cancer Res 16: 2634-2645, 2010.

19. Serrano MJ, Lorente JA, Delgado Rodríguez M, Fernández A, Fernández M, de la Torre C, Fernández Izquierdo J and Sánchez Rovira P: Circulating tumour cells in peripheral blood: potential impact on breast cancer outcome. Clin Trans Oncol 13: 204-208, 2011

20. Cristofanilli M, Budd GT, Ellis MJ, Stopeck A, Matera J, Miller MC, Reuben JM, Doyle GV, Allard WJ, Terstappen LW and Hayes DF: Circulating tumor cells, disease progression, and survival in metastatic breast cancer. N Engl J Med 351: 781-791, 2004.

21. Pachmann K, Camara O, Kavallaris A, Krauspe S, Malarski N, Gajda M, Kroll T, Jörke C, Hammer U, Altendorf-Hofmann A, et al: Monitoring the response of circulating epithelial tumor cells to adjuvant chemotherapy in breast cancer allows detection of patients at risk of early relapse. J Clin Oncol 26: 1208-1215. 2008.

22. Pachmann K, Camara O, Kohlhase A, et al: Assessing the efficacy of targeted therapy using circulating epithelial tumor cells (CETC): the example of SERM therapy monitoring as a unique tool to individualize therapy. J Cancer Res Clin Oncol 137: 821-828, 2011.

23. Meng S, Tripathy D, Frenkel EP, Shete S, Naftalis EZ, Huth JF, Beitsch PD, Leitch M, Hoover S, Euhus D, et al: Circulating tumor cells in patients with breast cancer dormancy. Clin Cancer Res 10: 8152-8162, 2004.

24. Aguirre-Ghiso JA: Models, mechanisms and clinical evidence for cancer dormancy. Nat Rev Cancer 7: 834-846, 2007.

25. Mani SA, Guo W, Liao MJ, Eaton EN, Ayyanan A, Zhou AY, Brooks M, Reinhard F, Zhang CC, Shipitsin M, et al: The epithelial-mesenchymal transition generates cells with properties of stem cells. Cell 133: 704-715, 2008 